\title{
Seed Priming with Salicylic Acid Improves Germination and Seedling Growth of Rice (Oryza sativa L.) under PEG-6000 Induced Water Stress
}

\author{
Prabhasmita Shatpathy ${ }^{1}$, Manoranjan Kar ${ }^{1}$, Sanat Kumar Dwibedi ${ }^{2 *}$ and \\ Abhiram Dash ${ }^{4}$ \\ ${ }^{1}$ (Plant Physiology), ${ }^{2}$ (Agronomy), ${ }^{3}$ (Agricultural Statistics), Orissa University of Agriculture \\ and Technology, Bhubaneswar, Odisha-751003, India \\ *Corresponding author
}

Keywords

Germination, Seedling growth, Deficit water stress, Seed priming, Salicylic acid

Article Info

Accepted: 10 September 2018 Available Online: 10 October 2018

\section{A B S T R A C T}

Seed germination and seedling establishment are most vital stages in plant growth cycle, playing major role in determining the final density of plants. In drought prone areas, poor seed germination and seedling emergence are the major problems. Seed priming is known to improve germination and seedling emergence under different environmental stresses. Salicylic acid (SA) also plays a major role in regulation of many physiological processes e.g. growth, development, ion absorption and germination of plants. An experiment was carried out in completely randomized design with three replications in the Plant Physiology laboratory of OUAT, Odisha in order to evaluate the effectiveness of seed priming with SA of $100 \mathrm{ppm}$ in improving seed germination, seedling vigor index and growth characteristics of rice grown under deficit water stress. Experimental treatments included 4 rice cultivars (Subhadra, Mandakini, Kalinga III and Khandagiri), 2 levels of seed priming (without SA and with SA of $100 \mathrm{ppm}$ ) and 5 levels of water stress, which were imposed by applying $0.0,-0.2,-0.4,-0.6$ and $0.8 \mathrm{MPa}$ osmotic solutions of Polyethylene Glycol (PEG)-6000. The results showed severe reduction in germination and seedling growth with increasing stress levels. Seed-priming with SA of $100 \mathrm{ppm}$ not only increased seedling dry weight but also reduced mean germination time compared to the untreated seeds. Seedling growth of SA-primed seeds had significantly higher root and shoot length than non-primed seeds. Such results indicated reduction in severity of the effect of water stress on germination and seedling growth parameters of rice by priming with SA of $100 \mathrm{ppm}$ which ultimately could tolerate deficit moisture conditions to some extent. Among the 4 test rice genotypes, the best alleviation of stress was achieved in the sensitive cv. Kalinga III. 


\section{Introduction}

Seed germination is one of the most important phases in the life cycle of plant which is highly responsive to its existing environment. It is one of the effective processes for enhancing the quality of cultivated plants which are widely consumed in the world. It gets affected severely under water limiting conditions and thus establishment of seedlings in dry environment is a major reason for seedling mortality. Food productivity is decreasing due to detrimental effects of various biotic and abiotic stresses and smothering of such stresses is the prime concern under changing climate. Drought, being the most important environmental stress, leads to a series of physio-morphological and molecular changes that severely impairs plant growth and development more than any other environmental factor (Shao et al., 2008; Shao et al., 2009 and Gamze et al., 2005). The success or failure of plant's establishment is determined by such a major physical parameter of an environment (Gamze et al., 2005). Generally, drought stress occurs when the available water in the soil is reduced and the atmospheric conditions cause continuous loss of water by transpiration or evaporation (KhajehHosseini et al., 2003). Available water resources for successful crop production have been decreasing in recent years. Furthermore, in view of various climate-change models scientists suggested that in many regions of world, crop losses due to increasing water shortage would further aggravate its impacts (Anjum et al., 2011).

Drought stress is primarily manifested as osmotic stress resulting in the disruption of homeostasis and distribution of ions in the cell (Wang et al., 2003). Under in vitro conditions, Polyethylene glycol (PEG), a non-ionic water polymer which is not expected to penetrate into plant tissue rapidly is widely used to induce water stress reproducibly (Macar et al.,
2009). As PEG does not enter into the apoplast, water is withdrawn from the cell including cell wall. Thus PEG solutions mimic dry soils in better way than the solutions of low-MR osmotica which infiltrate the cell wall with solution (Verslues et al., 1998).

The name 'salicylic acid' (SA) hales its origin from the Latin word "Salix", meaning willow tree. It is ubiquitously distributed in the whole plant domain (Raskin et al., 1990) and is classified under the group of plant hormones (Raskin, 1992). It has diverse controlling roles in the metabolism (Popova et al., 1997) with multitude of effects on the morphology and physiology of plants (Maghsoudia and Arvind, 2010). It also induces protective mechanism enhancing resistance to biotic and abiotic stresses (Zahra et al., 2010 and Szepesi et al., 2011) through regulation of antioxidantenzymes with the greatest role in stress condition in comparison to other hormones (Khan et al., 2003). Seed imbibition with SA or acetyl-SA, conferred to stress tolerance in plants, is more consistent with signaling for gene expression rather than their direct effects (Senaratna et al., 2000).

Therefore, given the importance of water stress at germination stage of rice (Oryza sativa L.), the objective of the current research was to study responses of its seeds and seedlings to deficit water stress and to investigate the possibility of mitigating the effects of water stress on it by pre-treating seeds with SA.

\section{Materials and Methods}

The current experiment was carried out in the plant physiology laboratory of the College of Agriculture, Orissa University of Agriculture and Technology (OUAT), Bhubaneswar, India during Kharif 2013. It was conducted in a completely randomized design (CRD) with 3 replications; 4 cultivars (Subhadra, 
Mandakini, Kalinga III and Khandagiri) of rice and 5 different levels of water potentials. The seeds and petridishes were sterilized by $10 \%$ hypochlorite solution and were thoroughly rinsed with sterile water. The sterilized seeds from each variety were divided into 2 sets. The $1^{\text {st }}$ and $2^{\text {nd }}$ sets of grains from each variety were separately soaked overnight in sterile-distilled water (control) or SA (100 ppm), respectively at $25^{\circ} \mathrm{C}$. Subsequently, seeds were removed and the surface was dried by using blotting paper. Osmotic solutions of $-0.2,-0.4,-0.6$ and -0.8 $\mathrm{MPa}$ water potentials were prepared by dissolving 11.8, 17.5, 21.2 and $25.4 \mathrm{~g}$ of PEG6000 in $100 \mathrm{~mL}$ of sterile-distilled water, respectively. The sterile-distilled water was used as control treatment. The primed seeds were again rinsed with sterile-distilled water and dried between two layers of blotting paper $\left(22^{\circ} \mathrm{C}\right.$ with relative humidity of $\left.60 \%\right)$. Only 50 pre-treated seeds of each cultivar in each treatment were allowed to germinate in petridishes on Whatman No. 1 filter paper. Each filter paper was saturated with the same volume of osmotic solutions of respective potentials and also with the sterile-distilled water (as control). The procedure was replicated thrice with 3 sets of such petridishes used for each variety. Petridishes were transferred to a germination chamber maintained at $25 \pm 1{ }^{\circ} \mathrm{C}$ and $60 \%$ relative humidity with $12 \mathrm{~h}$ day light. After $24 \mathrm{~h}$ (day 1 ), the seeds were checked on daily basis up to day 14 and the number of germinated seeds were recorded.

After taking out the seedlings from the petridishes, the hypocotyl and radicle lengths of 10 randomly selected seedlings from each replication were measured and were weighed to get fresh biomass. The 10 seedlings were then oven dried at $80{ }^{\circ} \mathrm{C}$ for $72 \mathrm{~h}$ and reweighed to measure the dry-biomass. The germination percentage (GP), seed vigor index (SVI), germination rate index (GRI), mean germination time (MGT), etc were also calculated by using the following equations.

Germination Percentage (GP): The GP was calculated using the formula; $\mathrm{GP}=\left(\mathrm{N}_{1} / \mathrm{N}_{2}\right) \mathrm{x}$ 100 (Shakirova et al., 2003). In this equation, $\mathrm{N}_{1}$ is number of germinated seeds and $\mathrm{N}_{2}$ is number of total seeds.

Seed Vigour Index (SVI): The SVI was calculated as; SVI $=$ (root length + shoot length) x GP

Germination Rate Index (GRI): The GRI was calculated by the formula; GRI $=\mathrm{G}_{1} / \mathrm{T}_{1}+$ $\mathrm{G}_{2} / \mathrm{T}_{2}+\ldots \ldots . .+\mathrm{G}_{\mathrm{n}} / \mathrm{T}_{\mathrm{n}}$ (Evetts and Burnside, 1973). In this equation, $G$ is germination count on a particular day and $\mathrm{T}$ is time interval (in days).

Mean Germination Time (MGT) in days: After final count, MGT was measured by the formula; $\mathrm{MGT}=\sum \mathrm{nD} / \sum \mathrm{n}$ (day) (Ellis and Roberts, 1981). In this equation, ' $n$ ' is the number of germinated seeds per day and D the number of days after sowing of seeds.

Germination Energy Percentage [GE (\%)]: Where, GE $(\%)=$ (Number of germinated seeds at 2 days after sowing / Total number of seeds tested) x 100 .

Mean Daily Germination (MDG): Where, $\mathrm{MDG}=$ Final GP/ number of days to final GP (Rubio-Casal et al., 2003).

Peak Value $(P V)$ : Where, PV = Final GP / No. Of days required to reach the peak value of germination (Czebator, 1962).

Germination Value $(G V)$ : Where, $\mathrm{GV}=\mathrm{PV} \mathrm{x}$ MDG (Czebator, 1962).

Statistical analyses: The data collected from the experiment relating to various germination parameters were analyzed in analysis of 
variance (ANOVA) technique as prescribed by Panse and Sukhatme (1985). The standard error of mean (S.Em \pm ) and the critical difference (CD) at $5 \%$ level of significance were calculated by the SPSS programme and statistical package.

\section{Results and Discussion}

\section{Germination percentage (GP)}

The germination count decreased with decrease in water potential or increase in osmotic potential of the growing medium (Table 1), but it was more pronounced in seeds without SA pre-treatment, which is consistent with the earlier findings of Murungu et al., (2004). All four cultivars of rice exhibited similar trend of germination but with variations among themselves. The seed germination was $100 \%$ irrespective of genotypes tested in control. The lowest GP was recorded in $-0.8 \mathrm{MPa}$ in both treated and untreated seeds and it reduced by $70.5 \%$ in non-treated seeds compared to the control. The effects of varieties, varieties $x$ SA priming interaction, varieties $\mathrm{x}$ moisture stress interaction, SA priming $\mathrm{x}$ moisture stress interaction and varieties $x$ SA priming $x$ moisture stress interaction at $\mathrm{P}=0.05$ were significant (Table 3). The mean GP in cv. Subhadra was the highest and in cv. Kalinga III it was the lowest (Fig. 1). Lower germination due to limited water uptake by the seeds was also reported by Dodd and Donovan (1999). The pre-treatment with SA improved the DP which corroborates the earlier findings of Shakirova et al., (2003) and Basra et al., (2006) who advocated for use of SA as germination inducer. Even at lower water potential (i.e. -0.6 and $-0.8 \mathrm{MPa}$ ), the GP significantly increased due to SA pretreatment in all the varieties. Interaction of SA and water potential on GP was found to be significant. It might be due to induction of antioxidant responses that protected the plants from dehydration damage (Singh and Usha, 2003; Hayat and Ahmad, 2007). The reason for decreasing germination with increasing level of moisture stress could be due to the decreased water potential and increased osmotic potential as mediated by solutedeveloped additive effect on the inhibition of seed germination. From present investigations, it is quite clear that seeds primed with SA of $100 \mathrm{ppm}$ proved to be effective in inducing stress tolerance at the germination stage in rice.

\section{Seed vigour index (SVI)}

The SVI decreased with increase in osmotic stress (Table 1) and the minimum SVI was at $0.8 \mathrm{MPa}$. Such reduction due to moisture stress was also observed by Das et al., (2005) in rice cultivars. The maximum SVI was observed in cv. Subhadra followed by cv. Mandakini, cv. Khandagiri and cv. Kalinga III in descending order (Fig. 1). The SVI increased significantly $(28.6 \%)$ with the application of SA. It was much more effective at $-0.8 \mathrm{MPa}$ than control. Seed pre-treatment with SA increased the SVI which is in agreement with the result of Khodary (2004) in maize plants. In hybrid rice, seeds with SA pre-treatment showed better germination pattern and higher vigour level than nontreated seeds under stress (Ruan et al., 2003). The SA presumably allowed some repairs of the damaged membrane caused by lower water potential. Nascimento and West (1998) indicated that the improvement in germination and vigour index of SA-primed seeds might be due to reserve mobilization of food material, activation and resynthesis of some enzymes, DNA and RNA.

\section{Germination rate index (GRI)}

With decrease in water potential, the GRI also decreased having the lowest value at $-0.8 \mathrm{MPa}$ irrespective of the varieties (Table 1). The 
GRI of non-treated seeds had decreased by $91.7 \%$ at $-0.8 \mathrm{MPa}$ as compared to the control $(0.0 \mathrm{MPa})$ which however decreased by $70.1 \%$ in the SA treated seeds. Among the cultivars, cv. Subhadra had the highest GRI followed by cv. Khandagiri, cv. Mandakini and cv. Kalinga III in descending order (Fig. 1). Pre-treatment with 100 ppm SA had substantially increased the GRI in the seeds by $21.5 \%$ as compared to the non-treated seeds irrespective of the testvarieties. The present result corroborated to the earlier findings of Yadavi et al., (2000), Jatai and Afzal (2001), Gholami et al., (2010) and Basu et al., (2004).

\section{Mean germination time (MGT)}

The mean time taken for the maximum germination expressed as MGT (days) increased in all the varieties with decrease in water potential (Table 2). The rate of increase was higher at lower water potentials and it was the highest at $-0.8 \mathrm{MPa}$ irrespective of 4 varieties. Among the varieties, cv. Kalinga III had taken the highest time (1.84 days) followed by cv. Mandakini (1.78 d), cv. Khandagiri (1.70 d) and cv. Subhadra (1.67 d) in reducing order attaining the maximum germination (Fig. 2). Pre-treatment of SA at $100 \mathrm{ppm}$ reduced the MGT by $24.4 \%$ in all the stress treated seeds irrespective of varieties compared to stress. The varietal and treatment differences were found to be statistically significant. Gamze (2005) in pea also observed decreased GP and increased GMT with increase of drought stress (due to PEG). The SA reduced GMT due to early starting of the germination processes in pre-treated seeds under stress condition and they emerged out of the soil earlier and established faster with lesser time exposed to pests and diseases to be splashing (Agarwal et al., 2005) and Arif (2005) also attributed the probable reason for such early emergence of the SA pre-treated seeds in soybean to the completion of pregermination metabolic activities making the seed ready for radicle protrusion compared to untreated seeds.

\section{Seedling growth}

The plumule and radicle length decreased in all the varieties with advancement of the moisture stress (Table 2). Although the rate of decrease was slightly gradual up to water potentials of $-0.4 \mathrm{MPa}$ but it stepped up thereafter.

Rice varieties responded differentially to the decrease in moisture regime with shoot and root growth. Overall, the cv. Kalinga III had the highest shoot length and cv. Subhadra the lowest under all water stress conditions except control. However, in all the non-treated seeds at $-0.8 \mathrm{MPa}$ there was no plumule initiation. Pre-treatment of seeds with SA increased the shoot length at $-0.8 \mathrm{MPa}$, but only in case of cv. Kalinga III and cv. Mandakini. Shoot growth was absent in cv. Subhadra and cv. Khandagiri even in SA application (Fig. 2).

On an average, SA increased the shoot length by $28 \%$ in the different varieties albeit significant varietal variations. The root length also decreased significantly with decrease in water potentials and such decrease was higher at lower water potential beyond $-0.4 \mathrm{MPa}$. The highest root growth was observed in cv. Subhadra $(2.73 \mathrm{~cm})$ followed by $\mathrm{cv}$. Mandakini $(2.10 \mathrm{~cm}), \mathrm{cv}$. Khandagiri $(2.08$ $\mathrm{cm})$ and the lowest was in cv. Kalinga III $(1.73 \mathrm{~cm})$ (Fig.2). The decrease in root length under stress at $-0.8 \mathrm{MPa}$ was $99.2 \%$ as compared to the control under un-treated condition but, SA treatment substantially increased the root length by $28.6 \%$, irrespective of varieties and water stress.

The cv. Subhadra produced the longest roots but shortest shoots among 4 varieties of rice under different levels of moisture deficit conditions. 
Table.1 Effect of salicylic acid on germination percentage (GP), seed vigor index (SVI) and germination rate index (GRI) of rice varieties under moisture stress

\begin{tabular}{|c|c|c|c|c|c|c|c|c|}
\hline \multirow{2}{*}{\multicolumn{2}{|c|}{ Variety }} & \multirow{2}{*}{$\begin{array}{c}\text { Stress } \\
(-\mathbf{M P a})\end{array}$} & \multicolumn{2}{|c|}{ GP } & \multicolumn{2}{|c|}{ SVI } & \multicolumn{2}{|c|}{ GRI } \\
\hline & & & $\begin{array}{l}\text { Without } \\
\text { SA }\end{array}$ & With SA & $\begin{array}{c}\text { Without } \\
\text { SA }\end{array}$ & With SA & $\begin{array}{l}\text { Without } \\
\text { SA }\end{array}$ & With SA \\
\hline \multirow{6}{*}{\multicolumn{2}{|c|}{ Subhadra }} & Control & 100.00 & 100.00 & 1074.67 & 1228.67 & 35.00 & 35.00 \\
\hline & & 0.2 & 100.00 & 100.00 & 411.67 & 572.67 & 31.76 & 32.42 \\
\hline & & 0.4 & 88.06 & 100.00 & 163.81 & 249.33 & 25.05 & 31.98 \\
\hline & & 0.6 & 86.40 & 92.15 & 63.79 & 117.75 & 19.43 & 27.55 \\
\hline & & 0.8 & 25.21 & 51.66 & 2.52 & 14.71 & 4.63 & 12.97 \\
\hline & & Mean & 79.94 & 88.76 & 343.29 & 436.63 & 23.17 & 27.98 \\
\hline \multirow{6}{*}{\multicolumn{2}{|c|}{ Mandakini }} & Control & 100.00 & 100.00 & 826.67 & 1010.67 & 35.00 & 35.00 \\
\hline & & 0.2 & 100.00 & 100.00 & 667.67 & 751.00 & 30.37 & 31.27 \\
\hline & & 0.4 & 54.25 & 86.66 & 44.68 & 178.53 & 25.52 & 26.98 \\
\hline & & 0.6 & 56.18 & 77.15 & 24.13 & 95.58 & 14.17 & 17.69 \\
\hline & & 0.8 & 8.55 & 51.88 & 0.26 & 31.19 & 2.48 & 5.61 \\
\hline & & Mean & 63.80 & 83.14 & 312.68 & 413.39 & 21.51 & 23.31 \\
\hline \multirow{6}{*}{\multicolumn{2}{|c|}{ Kalinga III }} & Control & 100.00 & 100.00 & 1022.67 & 1182.00 & 34.25 & 35.00 \\
\hline & & 0.2 & 100.00 & 98.55 & 409.00 & 574.17 & 32.67 & 34.13 \\
\hline & & 0.4 & 67.33 & 88.33 & 26.81 & 163.62 & 9.83 & 24.89 \\
\hline & & 0.6 & 54.51 & 71.33 & 16.35 & 66.50 & 11.44 & 20.49 \\
\hline & & 0.8 & 12.85 & 27.36 & 0.13 & 5.47 & 1.32 & 14.94 \\
\hline & & Mean & 66.94 & 77.11 & 294.99 & 398.35 & 17.90 & 25.89 \\
\hline \multirow{6}{*}{\multicolumn{2}{|c|}{ Khandagiri }} & Control & 100.00 & 100.00 & 1019.00 & 1117.67 & 34.49 & 35.00 \\
\hline & & 0.2 & 98.55 & 100.00 & 516.58 & 644.00 & 32.14 & 33.01 \\
\hline & & 0.4 & 77.15 & 91.40 & 65.48 & 129.67 & 22.11 & 25.59 \\
\hline & & 0.6 & 61.18 & 86.18 & 22.20 & 66.63 & 12.48 & 19.16 \\
\hline & & 0.8 & 15.91 & 42.36 & 0.32 & 2.12 & 3.09 & 8.41 \\
\hline & & Mean & 70.56 & 83.99 & 324.72 & 392.02 & 20.86 & 24.23 \\
\hline \multicolumn{3}{|r|}{ Total Mean } & 70.31 & 83.25 & 318.92 & 410.10 & 20.86 & 25.35 \\
\hline & & $\mathbf{P}$ & V & $P \times V$ & $\mathbf{T}$ & $\mathbf{P} \times \mathbf{T}$ & $\mathbf{V} \times \mathrm{T}$ & $P \times V \times T$ \\
\hline \multirow[t]{2}{*}{ GP } & SEm & 1.21 & 1.08 & 2.41 & 0.76 & 1.70 & 1.52 & 3.41 \\
\hline & $\begin{array}{l}\mathrm{CD} \text { a } \\
5 \%\end{array}$ & 3.39 & 3.03 & 6.78 & 2.14 & 4.80 & 4.29 & 9.59 \\
\hline \multirow[t]{2}{*}{ SVI } & SEm & 7.99 & 7.14 & 15.98 & 5.05 & 11.30 & 10.10 & 22.59 \\
\hline & $\begin{array}{l}\mathrm{CD} \text { a } \\
5 \%\end{array}$ & 22.48 & 20.10 & 44.96 & 14.22 & 31.79 & 28.43 & 63.58 \\
\hline \multirow[t]{2}{*}{ GRI } & SEm & 0.19 & 0.17 & 0.38 & 0.12 & 0.27 & 0.24 & 0.54 \\
\hline & $\begin{array}{l}\text { CD a } \\
5 \%\end{array}$ & 0.54 & 0.48 & 1.08 & 0.34 & 0.77 & 0.69 & 1.53 \\
\hline
\end{tabular}


Table.2 Effect of salicylic acid on mean germination time (MGT), shoot length (SL) and root length (RL) of rice varieties under moisture stress

\begin{tabular}{|c|c|c|c|c|c|c|c|c|c|}
\hline \multirow{2}{*}{\multicolumn{2}{|c|}{ Variety }} & \multirow{2}{*}{\multicolumn{2}{|c|}{$\begin{array}{l}\text { Stress } \\
\text { (-MPa) }\end{array}$}} & \multicolumn{2}{|c|}{ MGT (day) } & \multicolumn{2}{|c|}{$\mathrm{SL}(\mathrm{cm})$} & \multicolumn{2}{|c|}{$\mathbf{R L}(\mathrm{cm})$} \\
\hline & & & & $\begin{array}{c}\text { Without } \\
\text { SA }\end{array}$ & $\begin{array}{c}\text { With } \\
\text { SA }\end{array}$ & $\begin{array}{c}\text { Without } \\
\text { SA }\end{array}$ & With SA & $\begin{array}{c}\text { Without } \\
\text { SA }\end{array}$ & With SA \\
\hline \multirow{6}{*}{\multicolumn{2}{|c|}{ Subhadra }} & & Control & 1.00 & 1.00 & 4.94 & 5.36 & 5.81 & 6.93 \\
\hline & & & 0.2 & 1.19 & 1.18 & 0.40 & 1.46 & 3.72 & 4.27 \\
\hline & & & 0.4 & 2.05 & 1.45 & 0.04 & 0.15 & 1.84 & 2.35 \\
\hline & & & 0.6 & 2.44 & 1.61 & 0.00 & 0.00 & 0.74 & 1.28 \\
\hline & & & 0.8 & 2.70 & 2.08 & 0.00 & 0.00 & 0.10 & 0.28 \\
\hline & & & Mean & 1.88 & 1.46 & 1.08 & 1.39 & 2.44 & 3.02 \\
\hline \multirow{6}{*}{\multicolumn{2}{|c|}{ Mandakini }} & & Control & 1.12 & 1.00 & 4.11 & 5.85 & 4.16 & 4.26 \\
\hline & & & 0.2 & 1.25 & 1.18 & 2.74 & 3.29 & 3.94 & 4.22 \\
\hline & & & 0.4 & 2.01 & 1.39 & 0.18 & 0.44 & 0.65 & 1.67 \\
\hline & & & 0.6 & 2.65 & 1.47 & 0.00 & 0.16 & 0.43 & 1.09 \\
\hline & & & 0.8 & 3.42 & 2.31 & 0.00 & 0.08 & 0.03 & 0.52 \\
\hline & & & Mean & 2.09 & 1.47 & 1.41 & 1.96 & 1.84 & 2.35 \\
\hline \multirow{6}{*}{\multicolumn{2}{|c|}{ Kalinga III }} & & Control & 1.06 & 1.00 & 6.20 & 7.51 & 4.03 & 4.31 \\
\hline & & & 0.2 & 1.13 & 1.05 & 1.60 & 2.21 & 2.49 & 3.61 \\
\hline & & & 0.4 & 1.95 & 1.72 & 0.12 & 0.31 & 0.29 & 1.54 \\
\hline & & & 0.6 & 3.03 & 2.11 & 0.10 & 0.17 & 0.20 & 0.76 \\
\hline & & & 0.8 & 3.01 & 2.33 & 0.00 & 0.10 & 0.01 & 0.10 \\
\hline & & & Mean & 2.04 & 1.64 & 1.60 & 2.06 & 1.40 & 2.06 \\
\hline \multirow{6}{*}{\multicolumn{2}{|c|}{ Khandagiri }} & & Control & 1.00 & 1.00 & 5.30 & 5.79 & 4.89 & 5.39 \\
\hline & & & 0.2 & 1.31 & 1.14 & 1.83 & 2.18 & 3.42 & 4.26 \\
\hline & & & 0.4 & 1.44 & 1.32 & 0.10 & 0.24 & 0.75 & 1.18 \\
\hline & & & 0.6 & 2.91 & 1.66 & 0.04 & 0.21 & 0.32 & 0.56 \\
\hline & & & 0.8 & 3.09 & 2.11 & 0.00 & 0.00 & 0.02 & 0.05 \\
\hline & & & Mean & 1.95 & 1.45 & 1.45 & 1.68 & 1.88 & 2.29 \\
\hline \multicolumn{4}{|c|}{ Total Mean } & 1.99 & 1.51 & 1.38 & 1.78 & 1.89 & 2.43 \\
\hline & & & $\mathbf{P}$ & V & $P \times V$ & $\mathbf{T}$ & $\mathbf{P} \times \mathbf{T}$ & $V \times T$ & $P \times V \times T$ \\
\hline \multirow[t]{2}{*}{ MGT } & SEm & & 0.03 & 0.03 & 0.06 & 0.02 & 0.04 & 0.04 & 0.09 \\
\hline & $\mathrm{CD}$ a & & 0.09 & 0.08 & 0.17 & 0.05 & 0.12 & 0.11 & 0.24 \\
\hline \multirow[t]{2}{*}{ SL } & SEm & & 0.04 & 0.04 & 0.09 & 0.03 & 0.06 & 0.05 & 0.12 \\
\hline & $\mathrm{CD}$ a & $5 \%$ & 0.12 & 0.11 & 0.24 & 0.08 & 0.17 & 0.15 & 0.34 \\
\hline \multirow[t]{2}{*}{$\mathbf{R L}$} & SEm & & 0.06 & 0.05 & 0.11 & 0.04 & 0.08 & 0.07 & 0.16 \\
\hline & $\mathrm{CD}$ a & & 0.16 & 0.14 & 0.32 & 0.10 & 0.23 & 0.20 & 0.46 \\
\hline
\end{tabular}


Table.3 Effect of salicylic acid on seedling fresh weight (SFW), seedling dry weight (SDW) and germination energy percentage (GEP) of rice varieties under moisture stress

\begin{tabular}{|c|c|c|c|c|c|c|c|c|}
\hline \multirow{2}{*}{\multicolumn{2}{|c|}{ Variety }} & \multirow{2}{*}{$\begin{array}{l}\text { Stress } \\
\text { (-MPa) }\end{array}$} & \multicolumn{2}{|c|}{ SFW (mg) } & \multicolumn{2}{|c|}{ SDW (mg) } & \multicolumn{2}{|c|}{ GEP } \\
\hline & & & $\begin{array}{c}\text { Without } \\
\text { SA }\end{array}$ & With SA & $\begin{array}{c}\text { Without } \\
\text { SA }\end{array}$ & With SA & $\begin{array}{c}\text { Without } \\
\text { SA }\end{array}$ & With SA \\
\hline \multirow{6}{*}{\multicolumn{2}{|c|}{ Subhadra }} & Control & 18.80 & 21.05 & 7.05 & 7.55 & 100.00 & 100.00 \\
\hline & & 0.2 & 4.50 & 8.60 & 4.05 & 5.10 & 96.67 & 96.67 \\
\hline & & 0.4 & 1.85 & 3.00 & 0.85 & 1.50 & 69.28 & 91.66 \\
\hline & & 0.6 & 0.75 & 0.90 & 0.55 & 0.60 & 67.37 & 86.19 \\
\hline & & 0.8 & 0.00 & 0.30 & 0.00 & 0.25 & 18.09 & 35.24 \\
\hline & & Mean & 5.18 & 6.77 & 2.50 & 3.00 & 70.28 & 81.95 \\
\hline \multirow{6}{*}{\multicolumn{2}{|c|}{ Mandakini }} & Control & 17.70 & 20.85 & 6.35 & 6.80 & 100.00 & 100.00 \\
\hline & & 0.2 & 8.25 & 12.20 & 5.20 & 5.40 & 98.34 & 90.24 \\
\hline & & 0.4 & 1.40 & 2.40 & 1.05 & 1.65 & 65.51 & 78.34 \\
\hline & & 0.6 & 0.00 & 1.30 & 0.00 & 0.75 & 49.23 & 50.24 \\
\hline & & 0.8 & 0.00 & 0.60 & 0.00 & 0.32 & 5.72 & 14.52 \\
\hline & & Mean & 5.47 & 7.47 & 2.52 & 2.98 & 63.76 & 66.67 \\
\hline \multirow{6}{*}{\multicolumn{2}{|c|}{ Kalinga III }} & Control & 13.00 & 13.33 & 4.75 & 5.02 & 96.67 & 100.00 \\
\hline & & 0.2 & 6.55 & 8.35 & 3.75 & 4.35 & 100.00 & 100.00 \\
\hline & & 0.4 & 0.65 & 2.60 & 0.45 & 1.20 & 31.19 & 70.00 \\
\hline & & 0.6 & 0.00 & 1.20 & 0.00 & 0.62 & 36.91 & 59.52 \\
\hline & & 0.8 & 0.00 & 0.00 & 0.00 & 0.00 & 2.85 & 41.66 \\
\hline & & Mean & 4.04 & 5.10 & 1.79 & 2.24 & 53.52 & 74.24 \\
\hline \multirow{6}{*}{\multicolumn{2}{|c|}{ Khandagiri }} & Control & 14.45 & 18.50 & 5.85 & 6.65 & 98.34 & 100.00 \\
\hline & & 0.2 & 6.50 & 8.00 & 3.60 & 4.55 & 95.24 & 98.34 \\
\hline & & 0.4 & 0.70 & 1.75 & 0.60 & 1.40 & 70.00 & 81.91 \\
\hline & & 0.6 & 0.34 & 1.10 & 0.25 & 0.75 & 49.52 & 78.81 \\
\hline & & 0.8 & 0.00 & 0.00 & 0.00 & 0.00 & 13.09 & 26.19 \\
\hline & & Mean & 4.40 & 5.87 & 2.06 & 2.67 & 65.24 & 77.05 \\
\hline \multicolumn{3}{|c|}{ Total Mean } & 4.77 & 6.30 & 2.22 & 2.72 & 63.20 & 74.98 \\
\hline & & $\mathbf{P}$ & $\mathbf{V}$ & $\mathbf{P} \times \mathbf{V}$ & $\mathbf{T}$ & $\mathbf{P} \times \mathbf{T}$ & $\mathbf{V} \times \mathbf{T}$ & $\underset{T}{P \times \mathbf{V} x}$ \\
\hline \multirow[t]{2}{*}{$\overline{\text { SFW }}$} & SEm & 0.12 & 0.10 & 0.23 & 0.07 & 0.16 & 0.15 & 0.33 \\
\hline & $\begin{array}{l}\mathrm{CD} \text { a } \\
5 \%\end{array}$ & 0.33 & 0.29 & 0.66 & 0.21 & 0.46 & 0.41 & 0.93 \\
\hline \multirow[t]{2}{*}{ SDW } & SEm & 0.04 & 0.03 & 0.08 & 0.02 & 0.05 & 0.05 & 0.11 \\
\hline & $\begin{array}{l}\mathrm{CD} \text { a } \\
5 \%\end{array}$ & 0.11 & 0.10 & 0.22 & 0.07 & 0.15 & 0.14 & 0.30 \\
\hline \multirow[t]{2}{*}{ GEP } & SEm & 0.99 & 0.89 & 1.99 & 0.63 & 1.41 & 1.26 & 2.81 \\
\hline & $\begin{array}{l}\mathrm{CD} \text { a } \\
5 \%\end{array}$ & 2.80 & 2.50 & 5.59 & 1.77 & 3.95 & 3.54 & 7.91 \\
\hline
\end{tabular}


Table.4 Effect of salicylic acid on mean daily germination (MDG), peak value (PV) and germination value $(\mathrm{GV})$ of rice varieties under moisture stress

\begin{tabular}{|c|c|c|c|c|c|c|c|c|}
\hline \multirow{2}{*}{\multicolumn{2}{|c|}{ Variety }} & \multirow{2}{*}{$\begin{array}{l}\text { Stress } \\
\text { (-MPa) }\end{array}$} & \multicolumn{2}{|c|}{ MDG } & \multicolumn{2}{|c|}{ PV } & \multicolumn{2}{|c|}{ GV } \\
\hline & & & $\begin{array}{c}\text { Without } \\
\text { SA }\end{array}$ & With SA & $\begin{array}{c}\text { Without } \\
\text { SA }\end{array}$ & With SA & $\begin{array}{c}\text { Without } \\
\text { SA }\end{array}$ & With SA \\
\hline \multirow{6}{*}{\multicolumn{2}{|c|}{ Subhadra }} & Control & 7.14 & 7.14 & 100.00 & 100.00 & 714.29 & 714.29 \\
\hline & & 0.2 & 7.14 & 7.14 & 55.55 & 54.80 & 396.81 & 391.43 \\
\hline & & 0.4 & 6.29 & 7.14 & 51.51 & 55.84 & 321.90 & 398.83 \\
\hline & & 0.6 & 6.17 & 6.58 & 7.89 & 16.48 & 48.82 & 108.56 \\
\hline & & 0.8 & 1.80 & 3.69 & 0.99 & 2.98 & 1.79 & 11.11 \\
\hline & & Mean & 5.71 & 6.34 & 43.19 & 46.02 & 296.72 & 324.84 \\
\hline \multirow{6}{*}{\multicolumn{2}{|c|}{ Mandakini }} & Control & 7.14 & 7.14 & 75.00 & 100.00 & 535.71 & 714.29 \\
\hline & & 0.2 & 7.14 & 7.14 & 66.66 & 75.00 & 476.17 & 535.71 \\
\hline & & 0.4 & 3.88 & 6.19 & 8.11 & 29.08 & 31.38 & 180.58 \\
\hline & & 0.6 & 4.01 & 5.51 & 5.58 & 19.60 & 22.44 & 108.63 \\
\hline & & 0.8 & 0.61 & 3.71 & 1.07 & 7.41 & 0.67 & 28.52 \\
\hline & & Mean & 4.56 & 5.94 & 31.28 & 46.22 & 213.27 & 313.55 \\
\hline \multirow{6}{*}{\multicolumn{2}{|c|}{ Kalinga III }} & Control & 7.14 & 7.14 & 70.00 & 100.00 & 500.00 & 714.29 \\
\hline & & 0.2 & 7.14 & 7.04 & 55.77 & 59.58 & 398.36 & 419.03 \\
\hline & & 0.4 & 4.81 & 6.31 & 9.29 & 12.87 & 44.65 & 80.85 \\
\hline & & 0.6 & 3.89 & 5.10 & 7.40 & 10.17 & 27.91 & 51.05 \\
\hline & & 0.8 & 0.92 & 1.95 & 4.69 & 9.05 & 4.22 & 17.56 \\
\hline & & Mean & 4.78 & 5.51 & 29.43 & 38.33 & 195.03 & 256.56 \\
\hline \multirow{7}{*}{\multicolumn{2}{|c|}{ Khandagiri }} & Control & 7.14 & 7.14 & 100.00 & 100.00 & 714.29 & 714.29 \\
\hline & & 0.2 & 7.04 & 7.14 & 62.50 & 62.50 & 439.44 & 446.43 \\
\hline & & 0.4 & 5.51 & 6.53 & 10.36 & 58.34 & 57.09 & 379.35 \\
\hline & & 0.6 & 4.37 & 6.16 & 8.23 & 11.94 & 35.95 & 73.51 \\
\hline & & 0.8 & 1.14 & 3.03 & 5.65 & 8.78 & 6.59 & 26.54 \\
\hline & & Mean & 5.04 & 6.00 & 37.35 & 48.31 & 250.67 & 328.02 \\
\hline & & $\begin{array}{l}\text { Total } \\
\text { Mean }\end{array}$ & 5.02 & 5.95 & 35.31 & 44.72 & 238.92 & 305.74 \\
\hline & & $\mathbf{P}$ & V & $\mathbf{P} \times \mathbf{V}$ & $\mathbf{T}$ & $\mathbf{P} \times \mathbf{T}$ & $\mathbf{V} \times \mathbf{T}$ & 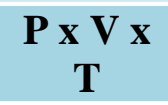 \\
\hline \multirow[t]{2}{*}{ MDG } & SEm & 0.09 & 0.08 & 0.17 & 0.05 & 0.12 & 0.11 & 0.24 \\
\hline & $\begin{array}{l}\text { CD at } \\
5 \%\end{array}$ & 0.24 & 0.22 & 0.48 & 0.15 & 0.34 & 0.31 & 0.69 \\
\hline \multirow[t]{2}{*}{ PV } & SEm & 0.93 & 0.84 & 1.87 & 0.59 & 1.32 & 1.18 & 2.64 \\
\hline & $\begin{array}{l}\text { CD at } \\
5 \%\end{array}$ & 2.63 & 2.35 & 5.26 & 1.66 & 3.72 & 3.32 & 7.43 \\
\hline \multirow[t]{2}{*}{$\mathbf{G V}$} & SEm & 6.42 & 5.74 & 12.85 & 4.06 & 9.08 & 8.12 & 18.17 \\
\hline & $\begin{array}{l}\mathrm{CD} \text { at } \\
5 \%\end{array}$ & 18.07 & 16.17 & 36.15 & 11.43 & 25.56 & 22.86 & 51.12 \\
\hline
\end{tabular}


Fig.1 Effect of Salicylic acid on germination percentage (GP), seed vigour index (SVI) and germination rate index (GRI) of rice genotypes under moisture stress
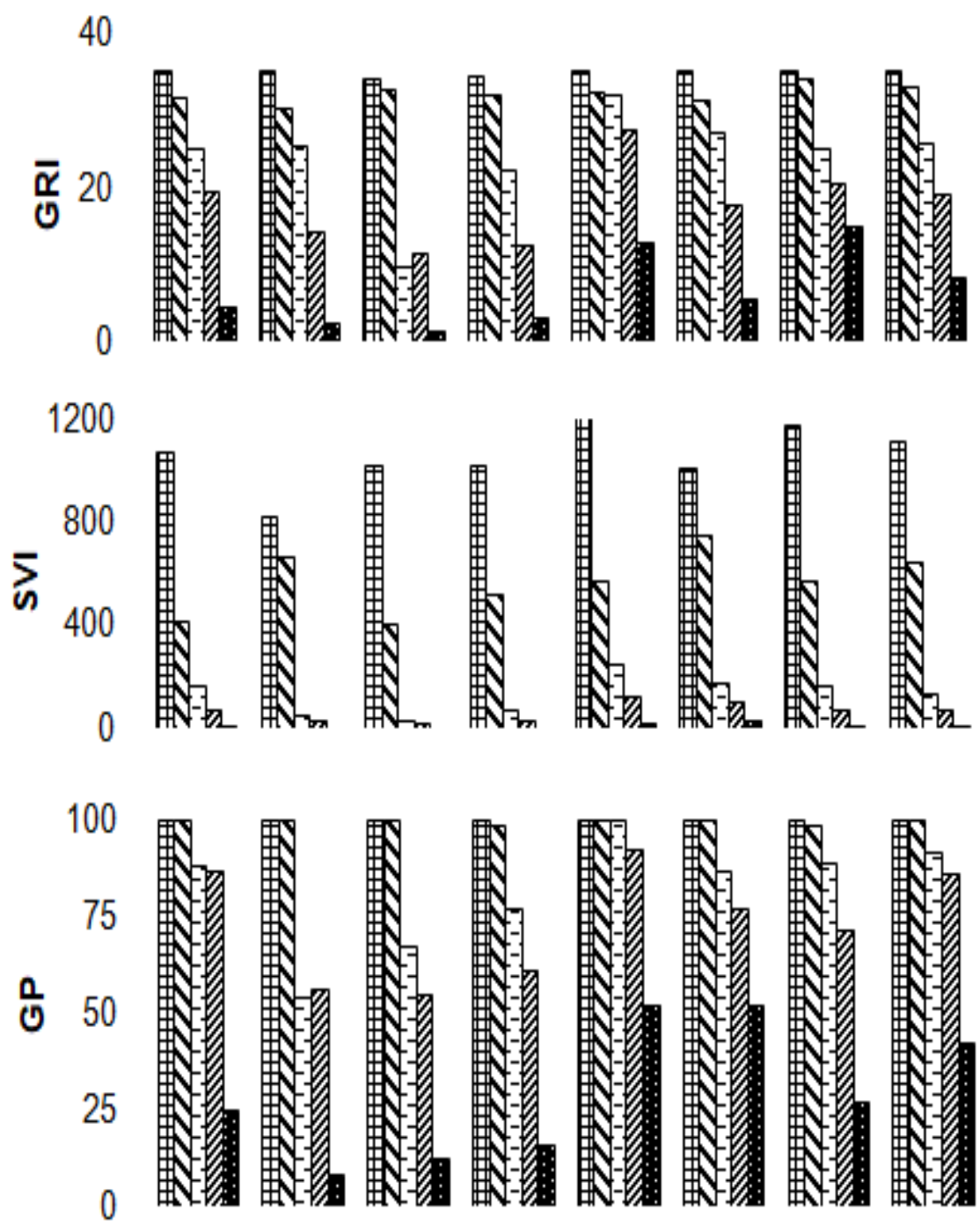

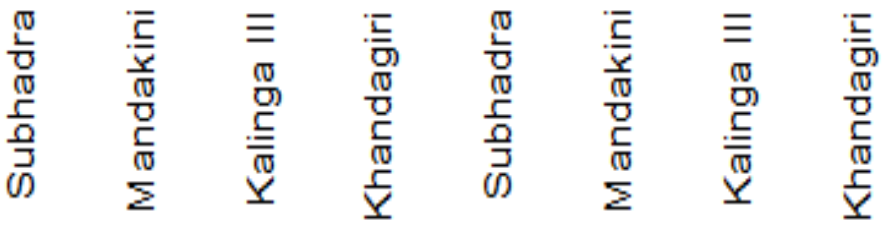

$$
\begin{aligned}
& 0.0 \mathrm{ppm} \quad 100 \mathrm{ppm}
\end{aligned}
$$

Salicylic acid concentration

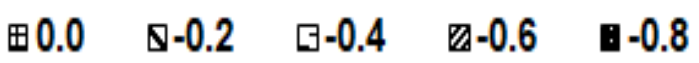


Fig.2 Effect of Salicylic acid on mean germination time (MGT), shoot length (SL) and root length (RL) of rice genotypes under moisture stress
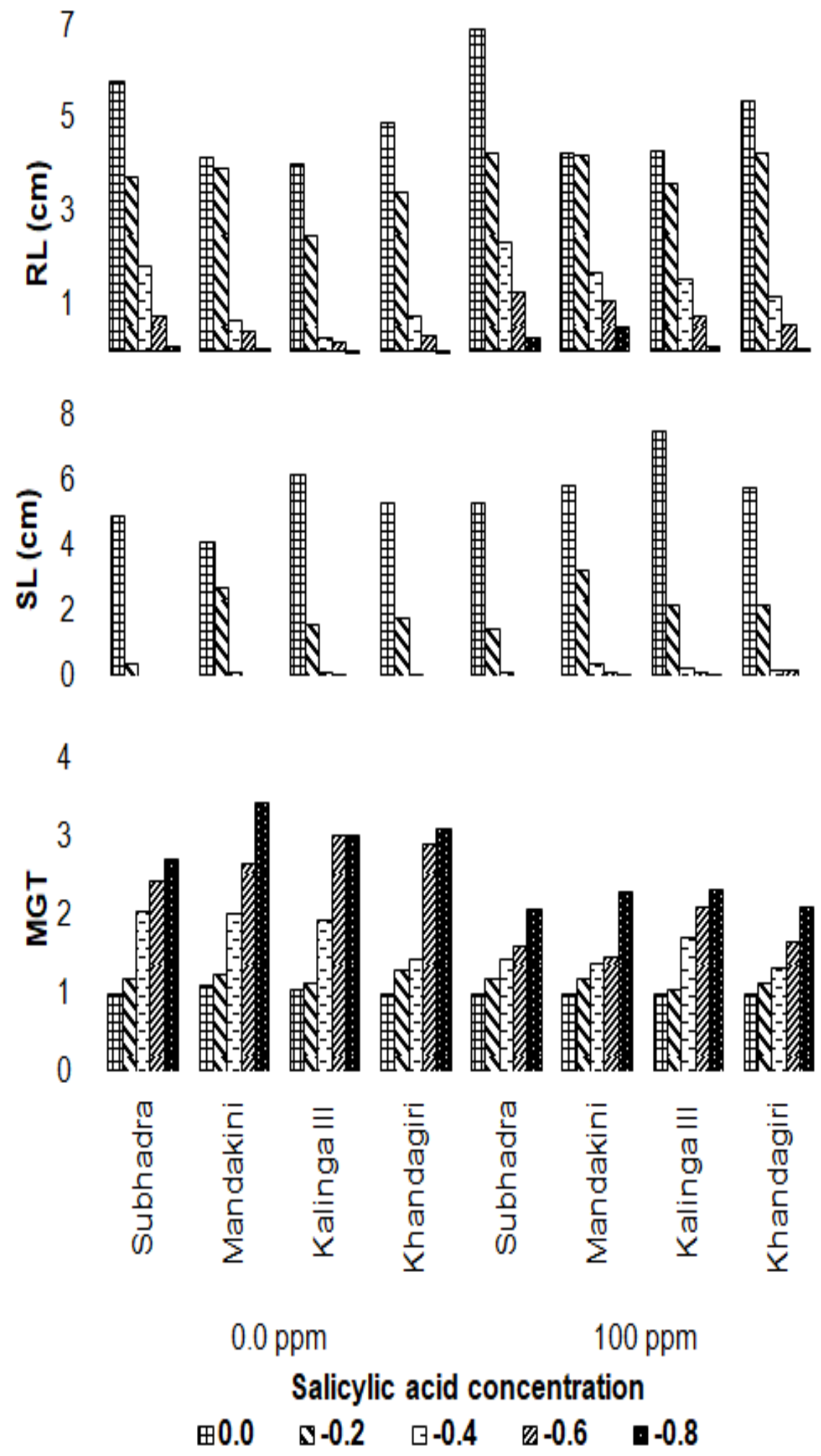
Fig.3 Effect of salicylic acid on Seedling fresh weight (SFW), Seedling dry weight (SDW) and Germination energy percentage (GEP) of rice varieties under moisture stress
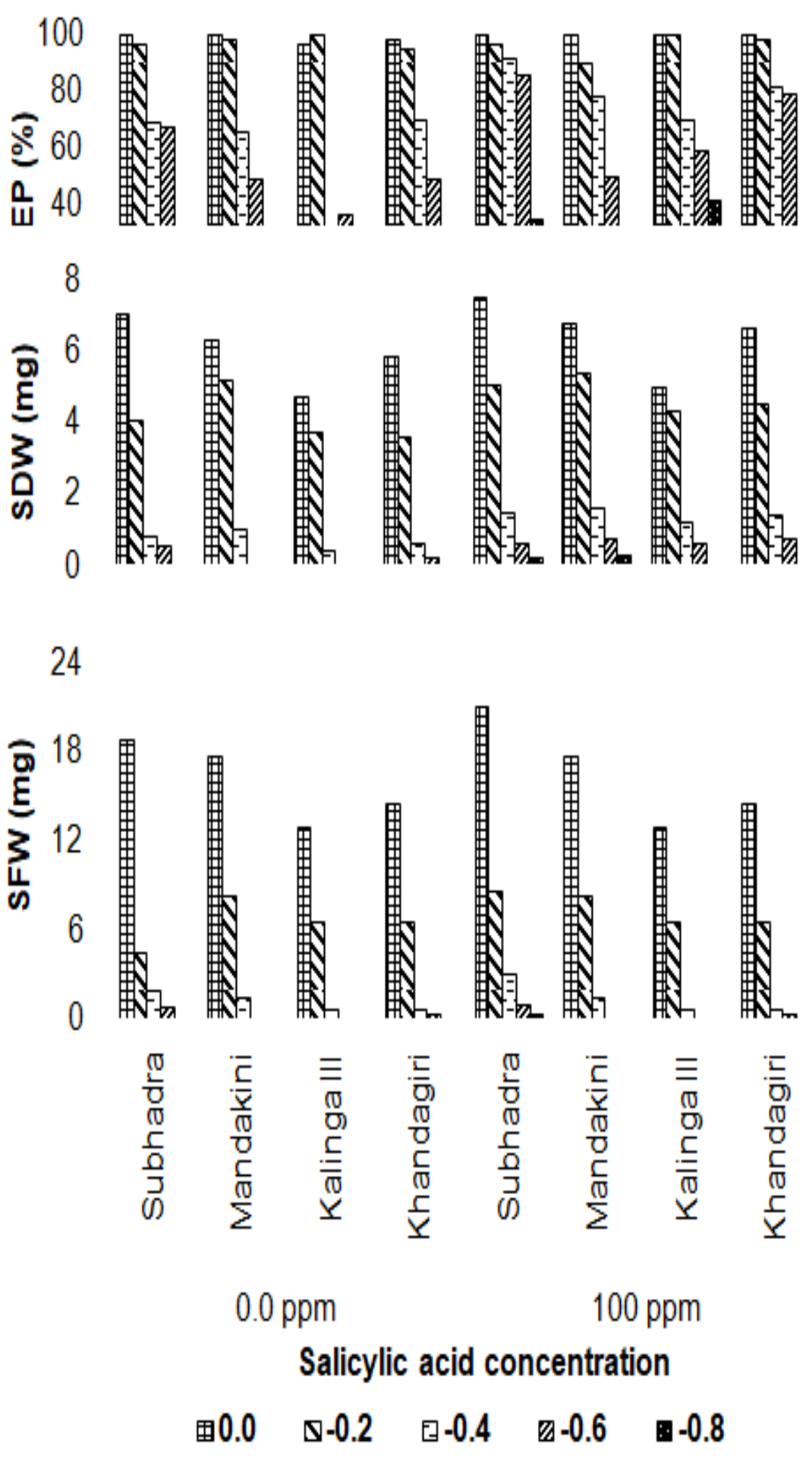
Fig.4 Effect of salicylic acid on Mean daily germination (MDG), Peak Value (PV) and Germination value $(\mathrm{GV})$ of rice varieties under moisture stress
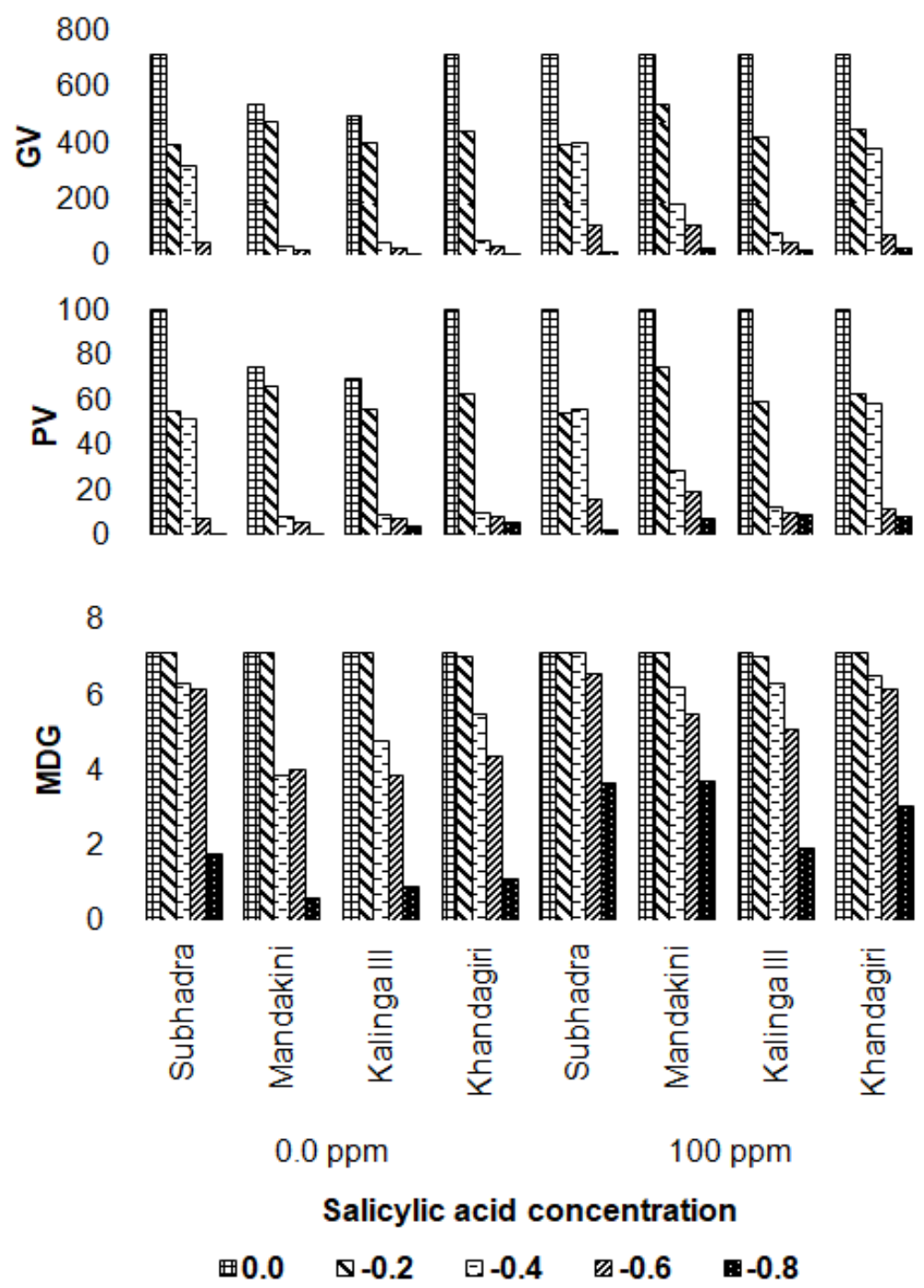

Thus, it could divert more of its dry matter to the roots than shoots on exposure to moisture stress. It was also evident that the tolerant varieties were more proficient in this aspect compared to less tolerant and susceptible varieties of rice. Werner and Finkelstein (1995) indicated that lower water potential slowed down water uptake by seeds, thereby inhibited their germination and root elongation. In this case, like GP, SA pretreated seeds had higher shoot and root length compared with un-primed seeds. The present result correlated with the earlier findings of
Hassanpouraghdam et al., (2009) in Brassica napus advocating positive effects due to the probable stimulatory effects of SA pretreatment on the early stages of germination process by mediation of cell division in germinating seeds.

\section{Seedling fresh weight (SFW)}

The fresh weight of seedlings decreased with the reduction in water potential (Table 3). Ultimately, at $-0.8 \mathrm{MPa}$, there was no shoot growth for all the varieties. Hence, very less 
or minimum seedling weight was obtained at $0.8 \mathrm{MPa}$. It was the heaviest in cv. Mandakini followed by cv. Subhadra, cv. Khandagiri and cv. Kalinga III (Fig. 3). On an average, the seedling fresh-weight increased by $32.1 \%$ with the application of SA (100 ppm). Since there was significant interaction between varieties $x$ SA priming $x$ moisture stress, the highest seedling fresh weight was relative to cv. Subhadra under control (PEG solution at $0.0 \mathrm{MPa})$ with priming $(21.05 \mathrm{mg})$ and the lowest seedling fresh weight was obtained under PEG solution at $-0.8 \mathrm{MPa}$ without priming in all 4 cultivars. Such results are in consonance with Hanan (2007) in wheat and barley.

\section{Seedling dry weight (SDW)}

The dry weight of seedlings decreased with the reduction of water potential (Table 3 ). On an average, it was found to be equal for $\mathrm{cv}$. Subhadra and cv. Mandakini (2.75 mg each) followed by cv. Khandagiri (2.37 mg) and cv. Kalinga III (2.01 mg) (Fig. 3). The seedling dry-weight increased by $22.5 \%$ with the application of $100 \mathrm{ppm}$ SA. The present findings were in conformity with Singh and Usha (2003), reporting increased dry mass of wheat seedlings with application of SA under deficit water stress. The protective and growth promoting effect of SA were presumably due to increased level of cell division within the apical meristem of seedling root, which caused an increase in plant growth. It is pertinent to mention here that SA has strong potential power, as a non-enzymatic molecule that helps to mitigate stress injury, water stress in particular, vis-à-vis sustenance of plants in dried ecosystem.

\section{Germination energy percentage (GEP)}

The GEP decreased with increase in osmotic stress, whereas it increased with pre-treatment of SA at $100 \mathrm{ppm}$ compared to untreated one, in all the four cultivars under consideration (Table 3). On an average, PEG solution at $0.8 \mathrm{MPa}$ significantly reduced GEP as compared with $(-0.0 \mathrm{MPa})$ distilled water. The GEP was significantly different among the rice varieties with the highest in cv. Subhadra (76.1) followed by cv. Khandagiri (71.1), cv. Mandakini (65.2) and cv. Kalinga III (63.8) (Fig. 3). Pre-treated seeds and nontreated seeds had shown GEP $75 \%$ and $63.2 \%$, respectively. The GEP had increased $18.6 \%$ in SA pre-treated seeds as compared to seeds without SA treatment. This was in consonance with the findings of Naderi et al., (2013) in wheat.

\section{Mean daily germination (MDG)}

This trait is an index of the velocity and acceleration of germination (Table 5). With the successive decrease in water potential ($\Psi \mathrm{w})$, there was concomitant decrease in MDG (percent $\mathrm{day}^{-1}$ ) in all 4 varieties of rice. The value was the lowest at $-0.8 \mathrm{MPa}$ (1.12) in case of untreated seeds. The overall mean value implied that MDG was the highest in cv. Subhadra (6.02) followed by cv. Khandagiri (5.52), cv. Mandakini (5.25) and lowest being recorded in cv. Kalinga III (5.14) (Fig. 4). Application of SA had visibly increased such values under stress condition. On the whole, it increased the value of MDG by $18.5 \%$ as compared to the stress and such increase could be attributed to oxidative stress management induced by simulating environment. This corroborates with the result of Sharafizad et al., (2013) in wheat.

\section{Peak Value (PV)}

A significant variation in peak value was observed to decline with the reduction in water potential (Table 4). On an average, the mean reduction in $\mathrm{PV}$ of non-treated seeds at $-0.8 \mathrm{MPa}$ was $96.4 \%$ as compared to control values. Cv. Subhadra had significantly higher 
PV (44.6) than other three genotypes and the cv. Kalinga III had the least (Fig. 4). Application of SA in stress increased the PV to the tune of $26.6 \%$ over the stress condition. When SA pre-treatment was induced, all 4 varieties showed full PV under control condition. The lowest mean was related to cv. Kalinga III under moisture stress. This result was in conformity with the findings of Farahbakhsh (2012) in fennel seeds.

\section{Germination value (GV)}

The GV decreased with the successive decrease in water potential (Table 4). The water stress had negatively affected the GV. Due to decrease in water potential, GV of untreated seeds had reduced drastically by $99.4 \%$ at $-0.8 \mathrm{MPa}$ as compared to control $(0.0 \mathrm{MPa})$. However, the SA helped in counteracting this negative effect and increased the $\mathrm{GV}$ value from 3.32 to 20.93 even at $-0.8 \mathrm{MPa}$. The highest $\mathrm{GV}$ was observed in cv. Subhadra (310.8) followed by cv. Khandagiri (289.4), cv. Mandakini (263.4) and cv. Kalinga III (225.8) (Fig. 4).

On the whole, the application of SA increased $\mathrm{GV}$ by $28 \%$ over the untreated seeds. This correlates with the findings of Metwally et al., (2003) in barley seedlings.

Analyzing the findings of the present investigation, it could be concluded that increase in deficit moisture stress level caused severe reduction in all the germination parameters in rice seeds and increase in the MGT. Pre-treatment of rice seeds with 100 ppm SA increased the moisture stress tolerance of rice seeds by overcoming the adverse effect of stress on stated parameters. The beneficial response of SA in ameliorating the adverse effect of water stress was more in sensitive genotype cv. Kalinga III. Therefore, it could be used as an ameliorant to alleviate the negative effects of drought injury in rice.
Based on the results of this study, inference could be drawn that deficit moisture stress severely reduced germination and rice plantlet growth. Pretreatment of rice seeds by 100 ppm SA considerably improved germination and plantlet growth in moisture stress condition and will increase the GV and GP. The plantlet establishment was faster and resistance against biological and nonbiological stress was more. Thus, priming of seeds reduced the severity of the effect of deficit moisture stress and the best alleviation of stress was achieved in the sensitive cv. Kalinga III.

\section{Conflict of interest}

There is no conflict of interest among the 3 authors.

\section{Ethical approval}

This article does not contain any studies with human participants or animals performed by any of the authors.

\section{ORCID}

https://orcid.org/0000-0003-2093-7397

\section{References}

Agarwal, S., Sairam, K.R., Srivastava, G.C., Aruna, T., and Meena, C.R. (2005). Role of ABA, Salicylic acid, calcium and hydrogen peroxide on antioxidant enzymes induction in wheat seedlings. Plant Science, 169: 559-570.

Anjum, S.A., Xie, X., Wang, L., Saleem. M.F., Man, C. and Lei, W. (2011). Morphological, physiological and biochemical responses of plants to drought stress. African Journal of Agricultural Research, 6: 2026-2032.

Arif, M. (2005). Seed priming with salicylic acid improves emergence, yield and 
storability of soybean. Ph.D. thesis in Agronomy. NWFP Agricultural University Peshawar, Pakistan.

Basra, S.M.A., Farooq, M., Wahid, A. and Khan, M.B. (2006). Rice seed invigoration by hormonal and vitamin priming. Seed Science and Technology, 34: 753-758.

Basu, S., Sharma, S.P. and Dadlani, M. (2004). Storability studies on maize (Zea mays L.) parental line seeds under natural and accelerated ageing conditions. Seed Science and Technology, 32: 239-245.

Czebator, F. (1962). Germination value: an index combining speed and completeness of pine seed germination. Forest Science, 8: 386-396.

Das, K., Pradhan, T., Ghosh, S. and Mishra, B.K. (2005). Evaluation of drought resistance characteristics of upland rice cultivars. Oryza, 42(2): 138-144.

Dodd, G.L. and Donovan, L.A. (1999). Water potential and ionic effects on germination and seedling growth of two cold desert shrubs. American Journal of Botany, 86: 1146-1153.

Ellis, R.A. and Roberts, E.H. (1981). The quantification of ageing and survival in orthodox seeds. Seed Science and Technology, 9: 373-409.

Evetts, L.L. and Burnside, O.C. (1973). Early root and shoot development of nine plant species. Weed Science, 21: 289291.

Farahbakhsh, H. (2012). Germination and seedling growth in un-primed and primed seeds of Fenel as affected by reduced water potential induced by PEG-6000. International Research Journal of Applied and Basic Sciences, 3(4): 737-744.

Gamze O., Mehmet D.K. and Mehmet A. (2005). Effects of salt and drought stresses on germination and seedling growth of pea (Pisum sativum L.).
Turkish Journal of Agriculture, 29: 237242.

Gholami, M., Rahemi, M. and Kholdebarin, B. (2010). Effect of drought stress induced by polyethylene glycol on seed germination of four wild almond species. Australian Journal of Basic and Applied Science, 4(5): 785-791.

Hanan, E.D. (2007). Influence of salicylic acid on stress tolerance during seed germination of Triticum aestivum and Hordeum vulgare. Advances in Biological Research, 1: 40-48.

Hassanpouraghdam, M.B., EmaratPardaz, J. and FarsadAkhtar, N. (2009). The effect of salicylic acid-priming on germination and seedling growth of Brassica napus L. under salinity conditions. Journal of Food Agriculture and Environment, 7(2): 620-622.

Hayat, S. and Ahmad, A. (2007). Salicylic acid: a plant hormone. Springer Publication.

Jatai, S.A. and Afzal, M. (2001). Seed deterioration study in pea, using accelerated ageing techniques. Pakistan Journal of Biology and Science, 4: 1490-1494.

KhajehHosseini, M., Powell, A.A. and Bingham, I.J. (2003). The interaction between salinity stress and seed vigor during germination of soybean seed. Seed Science Technology, 1: 715-725.

Khan, W., Prithviraj, B. and Smith D.L. (2003). Photosynthetic responses of corn and soybean to foliar application of salicylates. Journal of Plant Physiology, 160: 485-492.

Khodary, S.E.A. (2004). Effect of salicylic acid on the growth, photosynthesis and carbohydrate metabolism in saltstressed maize plants. International Journal of Agricultural Biology, 6: 5-8.

Macar, T. K., Turan, O. and Ekmekcd, Y. (2009). Effects of Water Deficit Induced by $\mathrm{PEG}$ and $\mathrm{NaCl}$ on Chickpea 
(Cicer arietinum) Cultivars and Lines at Early Seedling Stages, Gazi University Journal of Science, 22(1): 5-14.

Maghsoudia, K. and Arvind, M.J. (2010). Salicylic acid and osmotic stress effects on seed germination and seedling growth of wheat (Triticum aestivum L.) cultivars. Plant Ecophysiology, 2: 7-11.

Metwally, A., Finkmemeier, I., Georgi, M., and Dietz, K.J. (2003). Salicylic acid alleviates the cadmium toxicity in barley seedlings. Plant Physiology, 132: 272-281.

Murungu, F.S., Chiduza, C., Nyamugafata, P., Clark, L.J. and Whalley, W.R. (2004). Effects of on farm seed priming on emergence, growth and yield of cotton and maize in semi-arid area of Zimbawe. Experimental Agriculture, 40: 23-36.

Naderi, A., Sharafizad, M., Siadat, S.A., Sakinejad, T. and Lak, S. (2013). Effect of salicylic acid pre-treatment on germination of wheat under drought stress. Journal of Agricultural Science, $5(3)$.

Nascimento, W.M. and West, S.H. (1998). Priming and seed orientation affect emergence and seed coat adherence and seedling development of muskmelon transplants. HortScience, 33: 847-848.

Panse, V.G. and Sukhatme, P.V. (1985). Statistical methods for agricultural workers. ICAR Publications, New Delhi.

Popova. L., Pancheva, T. and Uzunova, A. (1997). Salicylic acid: Properties, biosynthesis and physiological role. Bulgarian Journal of Plant Physiology, 23: 85-93.

Raskin, I. (1992). Role of salicylic acid in plants. Ann. Rev. Plant Physiology and Plant Molecular Biology, 43: 439-463.

Raskin, I., Skubatz, H., Tang, W. and Meeuse, B. J. D. (1990). Salicylic acid levels in thermogenic and non- thermogenic plants. Annals of Botany, 66: 376-383.

Ruan, S.L., Xue, Q.Z. and Wang, Q.H. (2003). Physiological effects of seed priming on salt-tolerance of seedlings in hybrid rice (Oryza sativa L.). Scientia Agricultura Sinica, 36: 463468.

Rubio-Casal, A.E., Castillo, J.M., Luque, C.J. and Figueroa, M.E. (2003). Influence of salinity on germination and seeds viability of two primary colonizers of Mediterranean salt pans. Journal of Arid Environment, 53: 145-154.

Senaratna, T., Touchell, D., Bunn, E. and Dixon, K. (2000). Acetylsalicylic acid (Aspirin) and salicylic acid induce multiple stress tolerance in bean and tomato plant. Plant Growth Regulators, 30: 157-161.

Shakirova, F.M., Shakhbutdinova, A.R., Bezrukova, M.V., Fatkhutdionova, R.A. and Fatkhutdionova, D.R. (2003). Changes in the hormonal status of wheat seedling induced by salicylic acid and salinity. Plant Science, 164:317322.

Shao, H.B., Chu, L.Y., Jaleel, A.C. and Zhao, C.X. (2008). Water-deficit stressinduced anatomical changes in higher plants. Comptes Rendus Biologies, 331: 215-225.

Shao, H.B., Chu, L.Y., Jaleel, C.A., Manivannan, P., Panneerselvam, R. and Shao, M.A. (2009). Understanding water deficit stress-induced changes in the basic metabolism of higher plantsbiotechnologically and sustainably improving agriculture and the ecoenvironment in arid regions of the globe. Critical Review of Biotechnology, 29: 131-151.

Sharafizad, M., Naderi, A., Siadat, S.A., Sakinejad, T. and Lak, S. (2013). Effect of salicylic acid pre-treatment on germination of wheat under drought 
stress. Journal of Agricultural Science, $5(3)$.

Singh, B. and Usha, K. (2003). Salicylic acid induced physiological and biochemical changes in wheat seedlings under water stress. Plant Growth Regulator, 39: 137-141.

Szepesi, A., Gemes, K., Orosz, G., Peto, A., Takacs, Z., Vorak, M. and Tari, I. (2011). Interaction between salicylic acid and polyamines and their possible roles in tomato hardening processes. Acta Biologica Szegediensis, 55:165166.

Verslues, P.E., Ober, E.S. and Sharp, R.E. (1998). Root growth and oxygen relations at low water potentials, impact of oxygen availability in polyethylene glycol solutions. Plant Physiology, 116(4): 1403-1412.

Wang, W., Vinocur, B. and Altman, A. (2003). Plant responses to drought salinity and extreme temperatures towards genetic engineering for stress tolerance. Planta, 218(1): 1-14.

Werner, J.E. and Finkelstein, R.R. (1995).Arabidopsis mutants with reduced response to $\mathrm{NaCl}$ and osmotic stress. Physiology of Plant, 93: 659-666.

Yadavi, A., ModaressSanavi, A. and Zarghami, R. (2000). The effects of drought stress on oats species in germination step. Articles Summary in 6 Sessions of Agriculture and Plants Improvement Congress- Iran, Mazandaran University, pp: 235-236.

Zahra, S., Baghizadeh, A, B., Ali, V.S.M., Ali, Y. and Mehdi, Y. (2010). The salicylic acid effect on the tomato (Lycopersicum esculentum Mill.) sugar, protein and proline contents under salinity stress $(\mathrm{NaCl})$. Journal of Biophysics and Structural Biology, 2: $35-41$.

\section{How to cite this article:}

Prabhasmita Shatpathy, Manoranjan Kar, Sanat Kumar Dwibedi and Abhiram Dash. 2018. Seed Priming with Salicylic Acid Improves Germination and Seedling Growth of Rice (Oryza sativa L.) under PEG-6000 Induced Water Stress. Int.J.Curr.Microbiol.App.Sci. 7(10): 907924. doi: https://doi.org/10.20546/ijcmas.2018.710.101 